Gabriele Belletti

\title{
«-Scrivéma / scróiv ancàura». Il lavoro poetico di Giuliana Rocchi
}

\begin{abstract}
«È giusto che mi dia fastidio (la sera quando torno a casa) trovare la posta con su scritto "Alla poetessa" [...]. Non è la poetessa che gira tutto il giorno in mezzo al ferro vecchio, che accudisce a quattro uomini, che parla con la Rina, la mia cagnetta, con le galline i gatti [...] ma una povera diavola stanca, malridotta che avrebbe tanto bisogno di godersi in pace le trecentoottantamila lire di pensione di cinquant'anni e passa di lavoro».
\end{abstract}

(RocCHI 1986, 7)

\section{Abstract}

Il contributo prende in esame la poetica dell'autrice Giuliana Rocchi (Santarcangelo di Romagna, 1922 - Rimini, 1996), figura più appartata e meno nota rispetto agli altri poeti santarcangiolesi facenti parte del cosiddetto Circal de' giudéizi ("Circolo del giudizio"), come Raffaello Baldini e Tonino Guerra. In esso si ripercorrono sinteticamente le principali istituzioni delle sue raccolte dialettali, La vóita d’una dòna ("La vita di una donna", 1980), La Madòna di garzéun ("La Madonna dei garzoni", 1986) e il postumo Le parole nel cartoccio (1998). Si sottolinea in particolare come, grazie ad un'iniziale vocazione poetica ereditata dal padre analfabeta, l'autrice sia capace di tramandare in queste opere le voci e le storie di coloro che hanno abitato le contrade del suo paese natale, nel corso di una vita caratterizzata da difficoltà materiali, proteste per i diritti dei lavoratori e scontri con diverse forme di autorità e di potere. Da un lato, la poetessa critica i padroni e i politici, incapaci di comprendere le ragioni degli operai e delle persone comuni, denuncia il mancato rispetto per l'ambiente e per i luoghi naturali dell'infanzia dovuto al cambiamento dei tempi; dall'altro, con una vera e propria volontà di soccorso, canta le vicissitudini dei più fragili, siano essi esseri umani, cose o animali. Dall'analisi dell'opera dell'autrice si sono potute mettere in evidenza anche le sue riflessioni meta-poetiche, che restituiscono la lucida consapevolezza delle sue scelte stilistiche e del valore civile della poesia.

\footnotetext{
The e-journal «altrelettere» is hosted at the URL: http://www.altrelettere.uzh.ch , in accordance with the Open Access Policy of the University of Zurich. Please cite this article as follows: G. BELLETTI, «-Scrivéma / scróiv ancàura».Il lavoro poetico di Giuliana Rocchi, in «altrelettere», DOI: 10.5903/al_uzh-44.

(C) This article is licensed under a Creative Commons Attribution 2.5. Switzerland (CC BY-NC-ND 2.5). Please read the license terms on the website: http://creativecommons.org/licenses/by-nc-nd/2.5/ch/deed.en
} 


\section{Introduzione}

Col suo fiato autodidatta e orgogliosamente umile $_{1}$ - mai ingenuo (ARGNANI 2007, 17) 2 - tolto al susseguirsi della fatica del lavoro,3 ma da questo intrinsecamente dipendente, Giuliana Rocchi (Santarcangelo di Romagna, 1922 - Rimini, 1996) crea un'epica in versi, 4 distillata nelle sue opere scritte.5 La poetessa-operaia, corpo di fatica e poesia, tramanda le voci di coloro che hanno abitato, come lei non senza difficoltà, le contrade di Santarcangelo di Romagna. Tra queste, anche quella del padre analfabeta - Federico, detto Galinòun («A sò la fióla ad Galinòun», E’ mi viaz a Roma; "Sono la figlia di Galinòun", "Il mio viaggio a Roma”, III, 29) - che le ha fatto conoscere le satre,6 composte dalla Rocchi sin dal tempo di guerra ( «Ho cominciato in tempo di guerra, sul mio parente vedovo che si doveva sposar [...]. La prima seria l'ho fatta in corderia, quando abbiamo occupato la fabbrica» (MONTINI 1981)).7

La sua poesia in lingua madre («il dialetto, seconda lingua degli altri poeti dialettali di Santarcangelo [...], è la lingua prima» per lei, lingua che usa «con autorità assoluta» (MACRELLI 1981, 11)),8 viene pubblicata per la prima volta sui numeri di maggio, giugno e luglio del 19729 di «Tuttosantarcangelo», giornale locale, tuttora esistente, su cui scrivevano anche diversi membri del cosiddetto Circal de' giudéizi ("Circolo del giudizio").10 L’anno dopo, due suoi componimenti ${ }_{11}$ vengono letti - per iniziativa di Rina Macrelli Mel $_{2}$ - nel corso del seminario dedicato a Tonino Guerra, tenutosi presso la biblioteca cittadina, il 16 e 17 giugno 1973.13

La scrittura - non solo in forma lirica 14 - è pratica fondamentale per tramandare sia usanze, modi di dire («E' groèn [...] / l'è cmè una bèla dòna / nóv móis pu e’ parturéss / quant l'è la stasàun bóna», E’ groèn, "Il grano [...] / è come una bella donna / nove mesi e si sgrava / quand’è stagione buona”, "Il grano", II, 100) e filastrocche ${ }_{15}$ appartenenti a una comunità e a un territorio,16 sia eventi più individuali e privati. La voce abitata da altre voci («Puraza viva! la rógia la Rusina...», E’ mi ba tla pescheréa; "Vongole vive! strilla la Rosina...”, "Mio babbo in pescheria", ibid., 30), in un dialetto ospitante altri dialetti,17 nella sua forma scritta è capace di conservare una «mancanza di mediazione», vista come una vera e propria «dote» da Tullio de Mauro (BESSONE 2016).18 


\section{Infanzia e autorità}

Questa dote è propria a una bambina che, prima minuscola di fronte al mondo («e' bosc dla Zvana / l'éra una foresta / e me a set àn / cumè Pignicarèl», L'urzìn; "Il bosco della Giovanna / era una foresta / e io a sett'anni / come Mignolino", "L'orcino", ibid., 24),19 divenuta adulta, sa restituire in primis le voci e i silenzi dell'infanzia vissuta in povertà, denunciando le condizioni misere sue (Andéva ancòura a scóla; “Andavo ancora a scuola”, III, 128)20 e dei suoi coetanei di allora (La Madòna di garzéun; “La Madonna dei garzoni”, II, 27).

Il silenzio dei bambini di fronte agli adulti pare proteggere una verità poetica ed esoterica 'scambiata' tra di loro 21 («e i burdéll / il sa parchè», La mi madrèna; "E i bambini / lo sanno perché”, "L’altra madre”, ibid., 34), che, se conservata e difesa, può ancora, passati decenni, essere finalmente riferita e trasmessa.22 È proprio ricordando la verità dell'infanzia che la poetessa in $\mathrm{Al}$ ròbi brótti de nòst mònd ("Le cose brutte di questo mondo", III, 51) affida a un bambino «una gòmma culuroèda» ("una gomma colorata”) per cancellare tutte le cose brutte del mondo.23 La poesia nata, senza mai farsi «vittimistica» (MACRELLI 1981, 14), da un'esigenza di denuncia («laddove l’impulso della comunità e della solidarietà tra poveri si rivela impotente a rimettere il mondo sul giusto piede, subentra la scrittura»),24 espone necessariamente anche voci antagoniste, diametralmente opposte a quelle dei bambini, quelle dell'autorità.

Crudeli e quasi mai compassionevoli - anche se, come vedremo, esistono eccezioni - sono le voci del potere subìto. Le leggiamo, ad esempio, nella lirica I òt varbèl dla Marina ("Gli otto verbali della Marina”, II, 21-22) - dove un maresciallo inflessibile redarguisce una ragazzina quattordicenne («Ta n’é e' fanèl / e nè la pumidóra / ut maènca i fréni / e t'é ènca e' scat-féss / e pardipiò ta n'é gnénc la licénza»; "Non hai il fanale / né il fanalino dietro / ti mancano i freni / e la bici è a scatto fisso / e per di più non hai neanche la licenza", ibid., 21) che lavora per aiutare la famiglia numerosa ( «A iò òt fradél znìn / sti du tri péss e' vó déi e' nost magné»; "Ho otto fratellini / 'sti due tre pesci per noi sono il pane”, ibid., 22) - e in Bióigh (“Garzone”, ibid., 35) - in cui la padrona / reggitrice (l'“azdora”) dà ordini, inesorabile («u iè al bés-ci da guarnoè / bsògna andoè a zarloè, l'è àura ad sèmna»; “c'è le bestie da governare / e c'è da andare a preparar la terra, è ora di semina”, ivi). Raro caso in cui compare una risposta alla reggitrice è quello in cui un giovane nonno della Rocchi, sgridato per non 
lavorare "bene", le fa osservare che ha appena accompagnato il figlio a un carro merci in partenza per la guerra («C'l'apa pazinzia padràuna / incù l'è stoè un brót dè / a iò 'pena carg / t’un caramérci / che burdèl», Guèra de zdòt; “Abbia pazienza padrona / è stato un brutto giorno / ho appena caricato / su un carro merci / il figliolo", "Guerra del diciotto", ibid., 101). La poetessa, ereditando questo rispondere etico e necessario, si indigna anche per autorità più potenti e "lontane", i politici - è la televisione a portarli dentro la casa (Tribèuna polética; "Tribuna politica", ibid., 72) - parlando della sua condizione personale e della sua misera pensione («A m vlì própria avdài murói / sa chi du bócch ch'a m’ì spedói?», La mi pensiàun (1974); "Mi volete veder morire / mandandomi “ste due lire?", "La mia pensione (1974)", I, 94).

Compaiono altresì nelle liriche, come si diceva, rare autorità cittadine elogiate poeticamente per aver mantenuto le promesse, come in La Zèla nóva ("La nuova Cella", III, 160-62) o personalità che ordinano-consigliano a fin di bene, come il dottore che, in italiano, cerca di convincere la poetessa a farsi togliere un dente («Dimmi Rocchi cosa è stato? / Un cazzotto qui t’han dato?», «La radice è uncinata / incompleta è l'eruzione / qui ci vuole l'incisione», $E$ ' dént de giudóizi; "Il dente del giudizio", ibid., 37). L'italiano è riportato anche in altre occasioni, in dinamiche però conflittuali tra autorità e operai. Ordina in italiano infatti la voce del padrone che licenzia i lavoratori della corderia: «"Sono tutti licenziati!"».25

\section{Cambiamenti dimensionali}

La poesia permette di resistere anche a una delle più crudeli e inesorabili autorità, quella del tempo. Rintracciando e cantando oggetti-testimoni, essa riesce a salvare ricordi individuali e collettivi. La voce della Rocchi canta «il travaglio delle cose condannate al logorio incessante del tempo» (FuCCI 2007, 26), è vero e proprio strumento di opposizione a tale dimensione "ladra" («e' témp gagliòt / u s'à tólt tót al virtó», Gagliòt; "il tempo ladro / ci ha tolto ogni virtù”, “Ladro”, II, 56). Nella lirica A m'arcórd (“Mi ricordo”, ibid., 54-55), «la coèsa ad Brancalètt» ("la casa di Brancalètt") richiama alla memoria «i zug spèsa i paièr / la léssa zò me tenimoèn dla scoèla adfura / i filaróin dla Delcisa e dla Cuncèta / al sbacaroèdi dla Giulia / al cantarèli dla Speroènza / la 
bustarghéda...» (“i giochi dietro i pagliai / le scivolate sullo scorrimano della scala esterna / i filarini della Delcisa e della Concetta / le risate a cascata della Giulia / le focacce della Speranza / i brustolini...") e il lenzuolo mai terminato che «dórma / te fònd d'un bavóll» ("dorme / in fondo al baule"), nella lirica L'éultum ricoèm dla mi Nanda ("L'ultimo ricamo della mia Nanda", III, 101), protegge il ricordo di colei che lo stava ricamando, poco prima che la morte la cogliesse. Gli oggetti si fanno relitti resistenti a una temporalità feroce che muta le loro già fragili fatture e che li potrebbe far scomparire seduta stante se non fosse per la capacità salvifica dei versi.

Il cambiamento dei tempi - e della velocità del loro scorrere ( «La fóila la éra lònga / e agli òuri a n paséva», La zrèla; "La fila era lunga / e le ore non passavano mai”, "La carrucola”, ibid., 163) - ha influenzato la natura stessa di questa voce, che, soprattutto negli ultimi anni, si fa più faticosa, concisa e sprezzante. Il lamento nel passato - rintoccato dal Campanone - gravido sì di difficoltà ma anche di passioni, dolcezza e immaginazione, nel presente misurato da «svégli móti» ("sveglie mute", Al svégli móti, "Le sveglie mute", II, 107) - si tramuta in un rimprovero netto e senza appello. Soprattutto perché il cambiamento dei tempi ha ripercussioni evidenti ${ }_{26}$ non solo sulla poetessa e sulla sua quotidianità, ma anche sui bambini e la natura, costretti a subire $A l$ ròbi brótti de nòst mònd ("Le cose brutte di questo mondo", III, 51).27 Leggiamo la lirica Un gn'è piò (“Non ci sono più”, ibid., 179):

U n gn'è piò al fraschi e i bdóll

che ma la brèzza dl'oèlba i s'inchinéva.

U n gn'è piò la canèzza duvè che néun burdéll

andémmi a foè i nóst bsógn.

U n gn'è gnénca piò al bunoèghi

pini ad lumagóin ch'a racuiémmi

ne i rozlamérda chi s divartéva

a córr dri mal su pali.

Adès l'è tótt cimént e luci.

"Non ci sono più le frasche e i pioppi che alla brezza dell'alba s'inchinavano.

Non ci sono più le canne dove noi bambini

andavamo a fare i nostri bisogni.

Non ci sono più neanche le bonaghe 
piene di lumachini che noi raccoglievamo

né gli stercorari che si divertivano

a correre dietro alle loro palle.

Adesso è tutto cemento e luci”.

Il tempo di «cimént e luci» ("cemento e luci") ha invaso, spazzandole via, piccole presenze naturali con cui i bambini avevano un rapporto quotidiano. Il cemento del presente ha mutato le usanze e le forme («Ta n la cnòss piò / i la à fata granda, incatramoèda», La stroèda biènca de pòzz lòng; "Non la riconosci più / l'hanno allargata, asfaltata", "La strada bianca del Pozzo Lungo", ibid., 122), le luci artificiali hanno sostituito quella naturale («I à mèss di lampiéun zàl / chi sa ad teróizia», Lampiéun zàl; "Hanno messo dei lampioni gialli / che sembrano malati di itterizia”, “Lampioni gialli”, ibid., 158), eliminando tanto piccole quanto importanti sezioni del paesaggio individuale («Póch piò / d’un fazulètt / ad tèra / u n gn'è piò / i i à fat e' salgoè», E' mi órt; "Poco più / di un fazzoletto / di terra / non c'è più / gli hanno fatto il selciato", "Il mio orto", ibid., 106) e cittadino. Questa sensibilità ecologica 28 - presente in molta poesia degli ultimi anni - si estende anche al vicino mare (E' moèr, "Il mare", ibid., 95) che «sta murénd» perché «i fiómm i l’à invlenoè» ("sta morendo" perché "i fiumi l'hanno avvelenato"). Una natura di certo amata e difesa, senza però essere privata della sua indole «violentante» e maligna - subita da chi canta soprattutto ai tempi dell'infanzia.29

Così come non occulta l'indole ostile della natura, pur difendendola, la Rocchi non nasconde neppure alcuni lati positivi dei cambiamenti sopraggiunti nel presente, soprattutto se paragonati alle difficoltà del passato («A dògg trègg àn / insén sla Lucia ad Zoèn / a caminémi sò ma l'Éus / a còi agli érbi [...] Chi m'avéss dét / che a soent'àn / a li saréb andoè còi sla màchina / tal campagni ad Gambètla!», Progréss; “A dodici anni tredici / con la Lucia di Zoèn / camminavamo su all'Uso / a coglier erbe [...]. M'avessero detto / che a sessanta / sarei andata a coglierle in auto / nelle campagne di Gambettola!", "Progressi”, II, 93). Certe novità apparse nella vita quotidiana, possono portare aiuto («A vagh vérs e' cavdoèl / a m so permèssa e' lóss de telèfan / però l'è stoè sultoènt / par dmandoè aiéut», E'telèfan; "Vado verso la fine / mi sono permessa il lusso del telefono / ma è stato soltanto / per poter chiedere aiuto", "Il telefono", III, 
24) o affrancare dalle condizioni precedenti. Emblematica la poesia E mi paòis ("Il mio paese", ibid., 80-83), in cui la poetessa si fa portavoce di un riscatto dei poveri, non risparmiando critiche a coloro che, a Santarcangelo, avevano tenuto a distanza gli abitanti delle contrade («In piaza u i stéva i benestoènt / e ma néum póri ignuroènt, / perché a n'émmi la creènza, / i s tnéva sémpra in lontanoènza»; "In piazza abitavano i benestanti / e a noi poveri ignoranti, / poiché eravamo senza creanza, / ci tenevano a distanza”). Ora che i tempi sono cambiati «i córr tótt sò» (“corrono tutti su”) e considerano le contrade un luogo dall'«aria piò fóina» (“aria più fine”).

Altri mutamenti che traghettano dal passato al presente («i témp i n’è cmè próima», La Madòna di Garzéun; "I tempi non son più come una volta”, “ $L a$ Madonna dei garzoni”, II, 27), si leggono in poesie che si occupano di crisi economica («At che mònd c'as sém ardótt / u iè la crisi un pó per tótt», Crisi; “In che mondo ci siamo ridotti / c’è la crisi un po’ per tutti”, “Crisi”, ibid., 66) e, più di frequente, denunciano una perdita dolorosa entro i limiti individuali («A avéva i dìnt / um manchéva e’ poèn / adèss e' poèn us bóta véa / e i dìnt / i m’è casc tótt», Dìnt; “Avevo i denti / mi mancava il pane / ora il pane si getta / e i

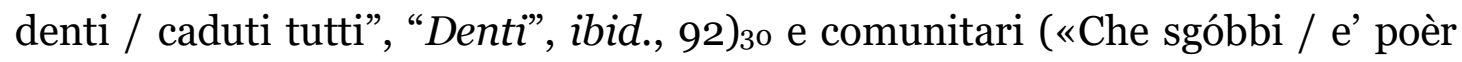
ch'e' séa pas e' teramòt / i à démolói tótt la furnoèsa. / U i éva lavuroè e' mi bà», Furnoèsa; "Che rabbia / sembra che sia passato il terremoto / hanno demolito la fornace. / Ci aveva lavorato il mio babbo", “Fornace”, III, 92).

I cambiamenti dei tempi non risparmiano neppure le lingue che si fanno così terreno di confronto tra generazioni. In Du sarài scàp, sti sacramìnt ("Dove saranno scappati, 'sti sacramenti?”, I, 85-88) compaiono infatti termini in un 'nuovo idioma', l'inglese, usati in un bar da giovani «cavléun» e «calzunaz» (“capelloni” e "calzonacci”). La Rocchi riesce ad 'accogliere' quei suoni stranieri nel dialetto santarcangiolese - "blue jeans" diventa «bludìnz» e "sandwich" «sènduic» - mentre riporta in discorso diretto il rimprovero di un vecchio del paese, meno predisposto forse alla comprensione e all'apertura («Scurói cum u v’à insgné la vostra mà [...] a vdirói che oènca i vécc i capirà»; "Parlate come mamma vi ha insegnato [...] e vedrete che anche i vecchi capiranno”, ibid., 88). 


\section{Fine e poesia}

Anche la morte - a cui nessuno può resistere, neppure i padroni («La vén / la $n$ guoèrda in faza / ma niséun / la tó sò tótt / vécc, zóvan, burdéll. / Ènca i padréun", La morta; "Viene / non ha riguardi / per nessuno / arraffa tutti / vecchi, giovani, bimbi. / Anche i padroni”, "La morte”, ibid., 30) e che la poetessa anziana sente inesorabilmente avvicinarsi («A sò arvata se cavdoèl / e par andoè bsògna avài moèl», La trombosi; "Sono arrivata al termine / e per andare bisogna avere male”, “La trombosi”, III, 127) - si manifesta “aggiornata” ai nuovi tempi. Prima, la condizione ultima veniva presagita, sapendo leggere come la nonna della Rocchi - particolari segni naturali, tramandati dalla tradizione, tra i quali il canto della civetta («Zétt zétt burdéll / andasói a lèt / prighé / e' coènta la zvèta / sàura la Ròca / e la vosta mà / la iè in coèva», La mi nòna Filóma; "Zitti, zitti, bambini / andate a letto / pregate / canta la civetta / sopra la Rocca / e vostra mamma / è alla fine”, “La mia nonna Filóma”, II, 23). Ora, anch'essa si è industriata, per mietere le sue vittime utilizza la mietilega meccanizzata («L’à pàuns la félza / l’à ciap / la mietiléga / e senza scavidé cal tendri / sa ógni érba / e' fa e' su fàs", Coèncar; "Ha posato la falce / ha preso / la mietilega / e senza tralasciare le tenere / d'ogni erba / fa un fascio", "Cancro", ibid., 95). Solo il cantarla può permettere, ancora, una qualche forma di resistenza e accettazione, servendosi anche di una certa dose di ironia. Una macabra ironia sulla morte la si legge, ad esempio, in La Rósa ad Camaroèn ("La Rosa di Camerano", ibid., 81), che «per quaiunoè la mórta / la tnéva la casa / sòta e' lèt; / e ogni toènt / la pruvéva» ("Per sfottere la morte / teneva la cassa / sotto il letto; / e ogni tanto / la provava”) e in Quant e' sarà òura (“Quando sarà il momento", III, 99), dove la poetessa chiede di non comprarle una cassa da morto nuova quando morirà, altrimenti «arvinarésvi tótt i dint mi vérmi // e mu me / i n m' u n rusgarébb piò» ("rovinereste tutti i denti ai vermi // e a me / non mi rosicchierebbero più”).

Resistere alla morte cantando significa anche esporre la propria commozione e compassione. Si può parlare di un'emozionata e materna partecipazione alle sofferenze di qualunque essere - umano, cosa o animale - non distante dall'idea di 'soccorso' in Anna Maria Ortese. ${ }_{31}$ La Rocchi, per la gattina morta in strada non nasconde le proprie lacrime («Sl’è vargògna un m’arimpórta / ò pianzéu / la mi gatina lan gnè piò / u m’è arvoènz / e' su litìn / sl'arvólt / e i rasp 
me còl», La mi gatina; "Se è vergogna non m’importa / ho pianto / la mia gattina non c'è più / mi è rimasto / il suo lettino / col risvolto / e i graffi nel collo”, "La mia gattina”, II, 84), per il barbagianni caduto dal nido non manca di criticare gli esseri umani che non sentono il suo lamento (Un vàul senza artàuran; “Un volo senza ritorno”, I, 119-120). La commozione e il soccorso si accompagnano alla riconoscenza per queste creature sofferenti o scomparse, come Kirina ("Kirina”, III, 65), la cagnetta da poco morta («Avéva sultoènt lì ch’la m vléva bén»; “Avevo solo lei che mi voleva bene”), o La Tóina ("La Tina”, ibid., 110), l'unica che «la éva lavoè i pan / ma la mi mà / tuberculòusa» (“aveva lavato i panni / alla mia mamma / tubercolosa”).

$\mathrm{Ai}$ versi escatologici si devono aggiungere anche alcune non secondarie riflessioni meta-poetiche della Rocchi, compiute nelle liriche stesse. Il suo non considerarsi poetessa ( «il suo, come diceva, era solo un bisogno di raccontare, di liberarsi, di ricordare, di far ricordare» (ROCCHI, 1998, 7)) 32 non esclude la cristallina consapevolezza del valore della poesia e della sua poesia ( $V$ Vést ch’im cèma dapartót / in sarà tót fagót / al pisrà stal zirudèli», E' bal di poéti; "Se mi chiamano dappertutto / non saranno tutti stupidi / piaceranno ste zirudelle”, "Il ballo dei poeti”, II, 60). L'idea che la poesia venga dalla vita o, per meglio dire, dopo la vita - e dopo il lavoro («Al mi poeséi / à l’ò sémpra détt / sono storie di vita vissuta», Ma la mi Rita; "Le mie non son poesie / io l'ho sempre detto / sono storie di vita vissuta", "Alla mia Rita", III, 59) 33 - che sia un privilegio a cui poche volte potersi concedere, si legge proprio nell'ultima lirica di II, quasi a giustificare la momentanea assenza di altri componimenti e la difficoltà di poterne scrivere di nuovi in futuro:

\section{Im da t'nèssa}

im dói da doèm da foè

che néun as n'andém

e i scrét i resta

ma pr'adès

l'è svóita la mi testa

ò véu trop da pansoè

m'um pó 'd niquèl.

E’scròiv 


\author{
"Mi stanno addosso \\ che mi dia da fare \\ che noi andiamo \\ e gli scritti rimangono \\ ma per adesso \\ la mia testa è vuota \\ ho avuto troppo da pensare \\ a un po' tutto". \\ "Lo scrivere" (II, 110)
}

Di grande rilevanza in tal senso, è anche la lirica Admoèn chilt ("Domani gli altri”, ibid., 87-88). Qui il momento escatologico incontra quello meta-poetico. Nino Pedretti, amico-poeta santarcangiolese, poco prima di morire, sprona la Rocchi a continuare a scrivere, soprattutto per coloro che poi leggeranno. È lei stessa a darne notizia nella lirica, come a dare valore e confermare l'accettazione di un così arduo compito ( «Scrivéma / scróiv ancàura / fin cu iè e’ témp / admoèn chilt i à da lèz / quel c'a lasém»; "Scriviamo / scrivi ancora / finché c'è tempo / gli altri dovranno leggere domani / ciò che lasciamo", ibid., 87-88). Solo la poesia nata dall'esperienza e dalla vita, può resistere alla morte - ma non solo, come abbiamo visto sopra - e accogliere, salvandoli, protagonisti, voci e ricordi di un'intera comunità.

\section{Conclusioni}

La scelta di focalizzare le nostre analisi sulla poetica di Giuliana Rocchi è strettamente connessa a quest'ultima consapevolezza e, in particolare, all'idea che grazie alla poesia dialettale sia possibile salvare l'anima di comunità che altrimenti rischierebbero di scomparire. Come sostiene Rebecca West, riferendosi all'opera del più noto Tonino Guerra, le poesie dialettali sono in grado di creare «an entire universe, at once vividly alive and gradually fading away, a world that lives and can only live in the language of its people, both of which - language and people - are part of an Italy quickly dying out as modernization and standardization eat away at the provincial and dialectal soul of the country» (WEST 1999, 12). Si tratta di una poesia tanto autentica quanto 
civile, che canta, come abbiamo rilevato, luoghi e persone, rispettando e difendendo la lingua in cui essi "vivono". Proprio per tali ragioni, dopo poco più di vent'anni dall'uscita della sua ultima opera, postuma, e in vista del centenario della sua nascita, ci pare importante continuare a indagare e salvaguardare il canto di questa poetessa - pregevoli in tal senso sono alcuni studi recentemente pubblicati e alcune iniziative del suo comune di origine, come l'istituzione di un Cantiere poetico dedicato alla poesia dialettale che ha visto la Rocchi protagonista nell'edizione del 2016, il ventennale della sua scomparsa. In un panorama ulteriormente stravolto dove "le cose brutte di questo mondo" hanno velocizzato a dismisura le proprie dinamiche e potenziato le proprie forze, dare attenzione agli ideali poetici di questa poetessa ci pare un atto doveroso e non rimandabile. $\mathrm{Ci}$ auguriamo pertanto venga presto alla luce una ristampa della sua opera completa - iniziata con la riedizione della sua prima raccolta nel 2016 - e la sua traduzione in altre lingue, traduzione capace, anche nel caso del dialetto, di trascendere i confini e aprire mondi (cfr. ibid., 20).

La vicenda letteraria di questa poetessa è emblematica, inoltre, perché la Rocchi è l'unica voce femminile ad accompagnare - senza far parte del summenzionato Circal de’ Giudéizi - quegli autori che hanno portato la poesia dialettale santarcangiolese alla ribalta, a partire dal secondo Novecento: Raffaello Baldini, Gianni Fucci, Nino Pedretti e lo stesso Tonino Guerra. Questo è un dato poetico non trascurabile, in particolar modo perché femminile è una delle voci più interessanti della nuova generazione di poeti provenienti dal paese abbarbicato sul Monte Giove, Annalisa Teodorani. L'eredità della Rocchi è stata da quest'ultima consapevolmente accolta, quasi con la stessa genuinità con cui la poetessa ha fatto sua la passione del padre. Una sorta di staffetta poetica e filiale che ci auguriamo possa essere oggetto anch'essa di ulteriori e necessari rilievi: «Presi in mano il suo primo libro [...], me ne attaccai come a una mammella e cominciai a scrivere di getto» (TEODORANI 2007, 27). 


\section{Note}

${ }_{1}$ Giuliana Rocchi inizia a lavorare senza aver terminato le scuole elementari, perché la madre Giuseppina muore quando lei ha soli dieci anni. Ciò non le impedirà di continuare da autodidatta la disciplina della propria voce poetica, con l'aiuto, come specificheremo, dell'amica Rina Macrelli. Una fulminea autobiografia la si legge nella lirica, datata 16 giugno 1973, $M a$ Tonino Guèra, contenuta nella sua terza raccolta, postuma, Le parole nel cartoccio (ROCCHI 1998, 43-45): «A sò noèda tla Cuntrèda / tra la zénta disagèda, / l'istruziòun a n la ò 'véuda / e pardunoè comm ch'a so 'vnéuda» ("Sono nata nelle Contrade / tra la gente disagiata, / l'istruzione non l'ho ricevuta / e perdonate come sono venuta", "A Tonino Guerra"). Tutte le traduzioni delle liriche riportate in questo contributo sono quelle contenute nelle opere citate. Per un'approfondita analisi della vita e della poetica dell'autrice rimandiamo a LisI 2018.

2 «Poetessa che non è per niente ingenua. Quindi leggendo attentamente la sua opera possiamo scoprire non solo una continua evoluzione linguistica, pur conservando saldo il senso istintivo dell'oralità, ma addirittura certi giochi verbali, certi scatti ritmici che scaturiscono in una autentica ricerca della parola», ARGNANI 2007, 17. Inoltre, la sua figura è di certo inseribile nel filone dei poeti popolari e attivi nelle piazze in Romagna tra la fine dell'Ottocento e gli inizi del Novecento, come Giustiniano Villa, Giovanni Montaldi e Bartoli di Bagnacavallo (cfr. BELLOSI 1994), pur distinguendosi per l'apporto più soggettivo ed esistenziale.

${ }_{3}$ La Rocchi svolge diversi lavori per tutto il corso della sua vita, senza mai rinunciare al lavoro poetico: dall'operaia in diverse fabbriche alla domestica. Lei stessa affermava: «Per quanto brutta ho anche la mia casa da guardare, oltre a quella degli altri. Ma le satre sono la mia passione», MACRELLI 1981, 10.

4 «La facoltà di narrare la "vita di una donna" per il tramite dei versi presuppone una comunità di ascoltatori e lettori che già condividano quelle parole e le capiscano nella loro dizione naturale, nella loro semantica. Vibra insomma qualcosa di epico nella Rocchi», DE SANTI 2007, 13 .

5 Trattasi delle raccolte La vita d'una dòna ("La vita di una donna", RocCHI 1981, da ora in avanti I), frutto di una lunga gestazione e pubblicata in una prima edizione nel 1980 per la casa editrice femminista Amanda; le già citate La Madòna di garzèun ("La Madonna dei garzoni", da ora in avanti II) e l'ultima raccolta, postuma, Le parole nel cartoccio (da ora in avanti III), che assembla testi inediti e versioni originali, da considerare il suo testamento poetico («'Sarà il mio testamento' - diceva ridendo», ARGNANI 2007, 18).

6 «Quelle che Giuliana Rocchi chiamava satre, cioè satire, un tipo di poesia popolare che gli antichi dicitori - tra cui il padre - immaginavano e coniavano di sana pianta esponendole oralmente nelle stalle, nelle aie o per le strade dei paesi, sono composizioni che le più soventi volte si raggrumano e si contraggono nello spazio di un evento significativo, qualche volta invece si estendono alla misura di un poemetto, dove le diverse invenzioni costruiscono un racconto o una sequenza di eventi. Ora, la struttura breve tende alla chiusura proverbiale, all'icasticità, quella più diffusa e larga racconta invece di avvenimenti che solcano trasversalmente il secolo», DE SANTI 2007, 11.

7 Intervista alla poetessa leggibile in MONTINI 1981.

8 In MACRELLI 1981, 11. Aggiungiamo, con Argnani, che «non è diminuire la qualità della sua arte il dire che Giuliana scrive come parla, perché lei parla solo la propria lingua madre, il dialetto di Santarcangelo, come l'acqua sgorga dalla roccia», ARGNANI 1989, 3.

9 Anno, lo ricordiamo, in cui esce anche la raccolta di Tonino Guerra, I bu ("I buoi") per le edizioni milanesi Rizzoli.

10 Rimandiamo a RicCI 2000.

${ }_{11}$ Le poesie in questione sono la già citata Ma Tonino Guèra ed E'vlén ("Il veleno", I, 72).

12 Amica dell'autrice, scrittrice e femminista, figura emblematica per la produzione e la trasmissione della poesia della Rocchi. Oltre a permetterle di essere conosciuta, Macrelli, prima lettrice, interviene direttamente nel suo laboratorio poetico - nelle prime due raccolte accompagnandola verso la stesura definitiva delle liriche (si vedano gli interventi sulla sintassi e sulla metrica). Si potrebbe quasi parlare di un lavoro svolto contemporaneamente da un soggetto interno al fare arte (Rocchi) e un soggetto esterno al fare in senso stretto, ma implicato direttamente nelle dinamiche della formazione della poesia (Macrelli). La poetessa lascia aperto il suo laboratorio e ne vuole condividere gli strumenti e i materiali con un soggetto-critico amico, di cui si fida e con cui vuole assolutamente e costantemente dialogare. 
${ }_{13}$ Vi partecipano, tra gli altri, Ignazio Buttitta, Augusto Campana, Tullio de Mauro, Friederich Schurr e Alfredo Stussi.

${ }_{14}$ La Rocchi scrive, infatti, ma molto raramente, anche in prosa. Ricordiamo i due racconti intitolati La Santoina de Ross ("La Santina del Rosso" - ripreso in versi nel poemetto Cum e, fótt ch'a m sò salvoè da la fòsa, "Come fu che mi sono salvata dalla fossa", I, 31-54) - e La camoisa ad Panòć ("La camicia di Panòce), entrambi contenuti in QUONDAMATTEO 1974.

${ }_{15}$ Rimandiamo, ad esempio, alla breve sezione dedicata alle filastrocche contenute in III, 143-49.

16 Una mappatura precisa, una micro-geografia del suo amato territorio - come per altri autori santarcangiolesi - si affaccia al lettore: si va dai fiumi (l'Uso, il Marecchia) ai monumenti (il Campanone, la Rocca), dalle città / cittadine vicine (Bellaria, Longiano, Viserba, Rimini) ai luoghi di lavoro (la Corderia).

${ }_{17} \mathrm{Si}$ ricordi che, come sostiene la curatrice di III, Rita Giannini, il dialetto della Rocchi non è statico ma «diverso, anche da una stesura all'altra, risentendo dell'influenza dei dialetti con cui era venuta a contatto, a cominciare da quelli dei suoi genitori, per ricomprendere quello riminese appreso durante il suo lavoro a Rimini e quello cesenate avvicinato prima durante il lavoro all'Arrigoni poi con il trasferimento quotidiano a Gambettola, in casa dei nipoti» (nella nota alla lirica Nina Nana, "Ninna nanna", III, 145-46).

18 BESSONE 2016.

19 Questo bosco viene citato anche nella lirica E' Pòzz Lòngh ("Il Pozzo Lungo", III, 123-24).

20 Qui la Rocchi canta la sua esperienza di bambina lavoratrice a soli «nóv dis an» ("nove dieci anni”).

${ }_{21}$ Raro caso in cui nei versi si 'sentono' dei bambini, è proprio quando essi comunicano tra di loro. Nell'ultima lirica della prima sezione di II, ad esempio, la Rocchi "fa sentire" la sua voce di bambina, la riporta in poesia, mentre si rivolge a un'amica d'infanzia: «-Dài sta sò menda disdài / s't'vén at pórt un óv da bài.-», «Um dól la poènza, a stag aquà, / dài Maria va tè piò in là», Ròbi da burdèli ("-Dài, sta su da lì a sedere / se vieni ti porto un uovo da bere -", "-Io ho mal di pancia, resto qua, / dài Maria, vai tu più in là", "Cose da bambine", II, 36).

${ }_{22}$ Il silenzio non significa che non vi sia una qualche forma di resistenza e di fermezza. $\mathrm{Si}$ vedano le due bambine che, per aver fatto appunto "cose da bambine", vengono punite e rimangono comunque impassibili: "Quant svimnédi ch'ém ciapoè / ma cmè t'un méur che l'avéss mnoè», Ròbi da burdèli ("Quante vincate ci siamo prese / ma era come se a un muro le desse", "Cose da bambine", II, 38).

${ }_{23}$ Sono solo i bambini ad avere «e giudiziin» (“il giudizio"): «T’è tri an / ad simpatéa / téni strétt / ch'i n vaga véa. // E giudiziin / ta l'è par trè / nu t fà granda / rèsta acsè», Ma la Camilla ("Hai tre anni / di simpatia / tienili stretti / che non scappino via. // Il giudizio / l'hai per tre / non ti far grande / rimani cosi”, “A Camilla”, III, 52).

${ }_{24}$ Postfazione di Gualtiero DE SANTI a III, 18.

${ }_{25}$ La batàia dla corderéa, (1964), "La battaglia della corderia (1964)”, ibid., 84-86. Un altro autore santarcangiolese, Nino Pedretti - che ritroveremo - legato alla Rocchi da reciproca stima e affetto, ha esplicitato il suo simile rapporto con l'italiano: «La lingua egemone è la lingua dell'impersonalità [...] quella anonimità del potere, della macchina derealizzante e burocratica, che sfrutta il linguaggio a uso coercitivo, che lo piega al servizio della propria imperscrutabilità», BERTOZZI 2007, 59. Vicinanze con la Rocchi si evincono anche nella considerazione del dialetto: «Il dialetto per me [...] serve all'artista per poter testimoniare di quello che egli ha veduto, e cioè la miseria fisica e morale, l'ingiustizia, la sofferenza collettiva delle classi oppresse nella guerra, nel lavoro, negli uffici, nelle fabbriche» (ibid., 58).

26 Lo si evince chiaramente leggendo la lirica Um buléva e' sangv ("Bollori”, II, 49): quando la poetessa aveva vent'anni anche se tutto era malandato e faceva freddo, il sangue bolliva; quando, in seguito, le condizioni materiali sono migliorate, quando è nella sua "térza giovinèza» ("terza gioventù"), ha freddo non più fuori, ma dentro, e il sangue è "marcio".

${ }_{27}$ Come riferisce in nota la curatrice Rita Giannini, la lirica già sopra citata è dedicata a Marco, un bambino di sei anni: «Lo spunto del titolo e della poesia Giuliana lo prese dall'allarmante notizia che riportava lo scoppio della centrale nucleare di Cernobyl» (cit.).

${ }_{28}$ Si vedano anche E'vlén (cit.) e I gréll, ("I grilli"), I, 106.

${ }_{29}$ Cfr. Tiziana MATTIOLI, Introduzione a III, XV.

зо De Santi sostiene in tal senso che il vettore della poesia rocchiana sia il corpo «di una donna che avverte e patisce l'indigenza, la fatica, e insieme sente le pulsioni dell'amore» (DE SANTI 2007, 12). Si legga, tra le altre, anche la lirica Al mi moèni ("Le mie mani", II, 108-9), dove le «moèni strachi» ("mani stanche") della poetessa dopo cinquant'anni di duro lavoro diventano oggetti attraverso cui si ricostruisce un'intera esistenza (lavorativa). 
${ }_{31}$ Rimandiamo in tal senso a ORTESE 2016.

${ }_{32}$ Note sulla raccolta della curatrice Rita GIANNINI, III, 7.

${ }_{33} \mathrm{Si}$ noti nel terzo verso ancora l'uso dell'italiano, quasi uno spiegare amplificato, per un pubblico più vasto, la natura dei suoi componimenti.

\section{Bibliografia}

\section{ARGNANI 1989}

Davide ARgnani, A proposito di Giuliana Rocchi, in «L'ortica», a. V, 5 (1989), settembre / ottobre, pp. 2-5.

ARGNANI 2007

Davide ARGNANI, Giuliana Rocchi: la linfa schietta della poesia, in «Il parlar franco. Rivista di cultura dialettale e critica letteraria», a. VII, 7 (2007), pp. 15-20.

BELLOSI 1994

Giuseppe BELlosi, I poeti di piazza in Romagna nel Novecento: tra oralità $e$ scrittura, in La poesia dialettale romagnola del '9oo, a c. di Gualtiero De Santi, Rimini, Maggioli Editore, 1994, pp. 57-69.

\section{BERTOZZI 2007}

Roberta BERTOZZI, Nei buchi che stanno tra le parole. Sulla poesia di Nino Pedretti, in «Il parlar franco. Rivista di cultura dialettale e critica letteraria», a. VII, 7 (2007), pp. 56-63.

BESSONE 2016

Vera BESSONE, "La poesia dialettale non è archeologia», intervista a Tullio De Mauro, in «Corriere di Romagna», 14 settembre 2016, articolo leggibile anche sulla pagina web del quotidiano: <http://www.corriereromagna.it/news/culturaspettacoli/19665/la-poesia-dialettale-non-e-archeologia.html > (07.06.2019).

DE SANTI 2007

Gualtiero DE SANTI, L'epica in versi di una vita, in «Il parlar franco. Rivista di cultura dialettale e critica letteraria», a. VII, 7 (2007), pp. 7-14.

FUCCI 2007

Gianni FUCCI, Vita e poesia (Un ricordo di Giuliana Rocchi), in «Il parlar franco. Rivista di cultura dialettale e critica letteraria», a. VII, 7 (2007), pp. 25-26.

LISI 2018

Cinzia LISI, La vicenda letteraria di Giuliana Rocchi, con introduzione di Tiziana Mattioli, Rimini, Maggioli Editore, 2018.

MACRELLI 1981

Rina MACRELLI, La storia fatta col corpo, in Giuliana Rocchi, La vita d'una dòna, a c. di Rina Macrelli, con introduzione di Tiziana Mattioli, Rimini, Maggioli Editore, 2a ed., 1981, pp. 5-18.

MONTINI 1981

Ileana MonTINI, Versi comperati al mercato, in «Il manifesto», 14 febbraio 1981.

ORTESE 2016

Anna Maria ORTESE, Le piccole persone, a c. di Angela Borghesi, Milano, Adelphi, 2016.

PAGLICCIA 2016

Fabio PAGLICCIA, Giuliana Rocchi: una voce popolare della poesia santarcangiolese, in «Letteratura e dialetti», a. XI, 9 (2016), pp. 63-81. 
QUONDAMATTEO 1974

E' viaz. Racconti e fiabe di Romagna, a c. di Gianni QuONDAMATTEO, con presentazione di Tullio De Mauro e tavole di Alberto Sughi, Imola, Galeati, 1974.

RICCI 2000

E' circal de' giudéizi. Letteratura. Santarcangelo nell'esperienza culturale del secondo dopoguerra, a c. di Manuela RICCI, introduzione di Renzo Cremante, Bologna, CLUEB, 2000.

RocCHI 1980

Giuliana RocchI, La vita d'una dòna, a c. di Rina Macrelli, con una nota di Vania Chiurlotto, Roma, Amanda, 1980.

RoCCHI 1981

Giuliana RocchI, La vita d'una dòna, a c. di Rina Macrelli, con introduzione di Tiziana Mattioli, Rimini, Maggioli Editore, 2a ed., 1981.

RocCHI 1986

Giuliana RocchI, La Madòna di Garzéun, a c. di Rina Macrelli, Rimini, Maggioli Editore, 1986.

RocCHI 1998

Giuliana RocchI, Le parole nel cartoccio, a c. di Rita Giannini, con postfazione di Gualtiero De Santi e testimonianze di Raffaello Baldini, Gianni Fucci e Tonino Guerra, Rimini, Maggioli Editore, 1998.

TEODORANI 2007

Annalisa TEODORANI, "E’ bén dabon": il mio ricordo di Giuliana Rocchi, in «Il parlar franco. Rivista di cultura dialettale e critica letteraria», a. VII, 7 (2007), pp. 27-28.

WEST 1999

Rebecca WeST, Introduzione a: Tonino Guerra, Abandoned Places, trad. inglese di Adria Bernardi, Toronto, Gernica, 1999, pp. 7-20. 